\title{
Stefan Marinov wins friends
}

\section{The suggestion that there are systematic departures from the strict requirements of special relativity has been persistently put forward by Dr Stefan Marinov. There is a case for repeating his experiment.}

STEFAn Marinov is a remarkable iconoclast who is convinced that Einstein's special theory of relativity is mistaken. Bulgarian by origin, Marinov has been, for all practical purposes, exiled from his homeland, but it is by no means clear whether his opinions on relativity or on the proper conduct of the Soviet leadership are chiefly responsible.

From his new home in neutral Austria, Marinov has been at loggerheads with several scientific journals, Nature among them, because of their refusal to publish any but a small part of his work, which now also includes a purported demonstration of a perpetuum mobile.

Marinov's frustrations with the journals have recently seemed to get the better of his judgement. Last year, angered by $\mathrm{Na}$ ture's refusal to publish a group of manuscripts, Marinov was for a time threatening to immolate himself in the square outside the British consulate at Genoa. Later, he threatened to do the same outside the British embassy in Vienna, but fortunately settled for a press conference instead. Those who have had dealings with Dr Marinov can have been left in little doubt that he is passionately convinced of the correctness of his position on special relativity. Quite apart from his apparent willingness to take his own life, it is clear that his waking hours are almost all spent in correspondence, much of it unfortunately fruitless, about this theory and about his purported demonstration, by means of what he calls the "coupled-shutters experiment", that the velocity of light is not, as Einstein's theory supposes that it would be, equal in all directions (isotropic) in all frames of reference.

Now, it seems, Marinov has won some influential friends. The issue of Physical Review Letters for 8 July $(55,143 ; 1985)$ contains an article by A.K. Maciel and J. Tiomno from the Brazilian National Science and Technology Research Council at Rio de Janeiro which at least puts Marinov's objections to special relativity in a context in which they can be grappled with. Moreover, what Maciel and Tiomno say is certain to have an important bearing on the classification of all possible experimental tests of special relativity.

Plainly, when there is such a mountain of experimental evidence that the general features of special relativity are borne out in practice, it would be foolish in the extreme to assert that special relativity is just plain wrong, thereby implying that the time has come to return to newtonian mechanics; after all, objects do not travel faster than light in any circumstances observable, while time dilation (as defined by Lorentz) is a fact of life and can be measured, for example, by the difference between the average lifetime of an unstable particle in motion and at rest. So if there are violations of special relativity, they are at most weak violations, departures from what is not strict orthodoxy and not flat contradictions of it.

But what weak violations of special relativity are neither trivial nor absurd? Borrowing from some work due to H.B. Ives in the 1930s, Maciel and Tiomno argue that it is prudent to disallow violations for electromagnetism, for independent particles in uniform motion and the other more elementary phenomena used as illustrations of special relativity, now even in the elementary textbooks. But what about the possibility that the behaviour of rigid bodies is not accurately accounted for by the Einstein-Lorentz transformations of coordinates, and that a rigid body remains rigid - the separation of fixed points remains constant - however it is moving?

The formal realization of this state of affairs seems not particularly elusive, although it is not possible to modify special relativity in any meaningful way without reintroducing the newtonian concept of absolute time and space, or the idea that there may be some preferred frame of reference such as that of the microwave background. Explicitly, if $x, y$ and $z$ are cartesian coordinates in that absolute frame of reference, and $t$ is the time, and if primed quantities are the same coordinates in a system moving at velocity $v$ along the $x$-axis, the transformed coordinates are exactly those of special relativity except for $t^{\prime}$, which is given (according to the formalism of Maciel and Tiomno) by $t=$ $\gamma t^{\prime}$, where $\gamma=\left(1-v^{2} / c^{2}\right)^{-1 / 2}$ and not the conventional Lorentz transformation in which $x v / c^{2}$ is added to $t^{\prime}$.

The calculations are not in themselves particularly difficult. It emerges simply enough that rigid rotating bodies remain rigid in the new system, and in the particular sense that the apparent angular velocity of all fixed points is a constant (if the angular velocity of the whole object is constant). The velocity of light is isotropic in the absolute system against whose coordinates the rotation of the rigid object is defined, while both time dilation and the Lorentz-Fitzgerald contraction (of lengths in the direction of relative motion) persist. What is sacrificed is the isotropy of the velocity of light in the rotating system.

The interest of what Maciel and Tiomno have done is that they can sort the several published tests of special relativity by the likelihood that they will yield a stringent test of the weakly violated form of special relativity defined by their transformation. The classical test of special relativity first carried out by Michelson and Morley and since repeated several times does not meet the test. The version of the experiment due to Joos (published in 1930) in which a Michelson interferometer was rotated on its turntable once every ten minutes or so, classically one of the better verifications of the isotropy of the velocity of light (among two orthogonal directions), will not suffice, although a more rapidly rotating turntable might meet the need. The simulation of the Michelson-Morley experiment in which two hydrogen masers are used as the source of radiation moving in orthogonal directions offers more hope of providing a stringent test of the modified transformation equations. And so, Maciel and Tiomno say, does Marinov's rotatingshutters experiment.

The principle of that is simple enough, a kind of Fizeau measurement of the velocity of light except that the objective is to make a direct comparison of the velocity of light in two opposite directions. Two disks with identical perforations are mounted parallel to each other at opposite ends of an axle and light from a laser is directed (by means of a beam-splitter) through the perforations of the disks in each direction. Marinov claims that his results, most recently obtained with home-made equipment at Graz, demonstrate that the velocity of light is not the same in all directions. He even claims to have been able to detect the velocity and direction of the Earth's movement through absolute space and time.

None of this proves that there is anything wrong with special relativity. It is merely a pointer to the kinds of tests that would be necessary to demonstrate a particular (and "weak") violation thereof. Maciel and Tiomno merely say of the Marinov experiment (as of the others on their list) that it "should be repeated even if to prove it wrong". It will be interesting to see how many correspondents write in to claim that that has been done already.

John Maddox 